\title{
Fluvastatin-induced alterations of skeletal muscle function in hypercholesterolaemic rats
}

\author{
Fuzi $\mathrm{M}^{1}$, Palicz $\mathrm{Z}^{1}$, Vincze $\mathrm{J}^{1}$, Cseri $\mathrm{J}^{1}$, Szombathy $\mathrm{Z}^{2}$, Kovacs $\mathrm{I}^{2}$, Olah $\mathrm{A}^{3}$, Szentesi $\mathrm{P}^{1}$, Kertai \\ $\mathrm{P}^{4}$, Paragh $\mathrm{G}^{5}$, Csernoch $\mathrm{L}^{1}$
}

from the

${ }^{1}$ Department of Physiology, ${ }^{3}$ Department of Clinical Biochemistry and Molecular Pathology, ${ }^{4}$ Department of Preventive Medicine and ${ }^{5}$ First Department of Medicine, MHSC, University of Debrecen, Debrecen, Hungary

${ }^{2}$ Department of Pathology, Kenézy Hospital LTD, Debrecen, Hungary

Running title: Statin and skeletal muscle

Address all correspondence to:

Dr. László Csernoch

Department of Physiology

University of Debrecen

P.O. Box 22

Hungary

H-4012 


\begin{abstract}
Although statins, the most widely used drugs in the treatment of hyperlipidaemia, are generally accepted as efficient and safe drugs their side-effects on skeletal muscle have been reported with increasing frequency in the past years. The lack of an appropriate animal model in which these side effects would consistently be observed is one of the most important drawbacks in studying statin associated myopathy. To overcome this and enable the studying of the effects of fluvastatin on skeletal muscles an animal model with high blood cholesterol levels was developed. In these animals cholesterol levels rose more than seven fold (from $1.5 \pm 0.1$ to $10.7 \pm 2.0 \mathrm{mmol} / \mathrm{L} ; \mathrm{n}=15$ and 16 ) with a dramatic increase in LDL/HDL ratio (from $0.29 \pm 0.02$ to $1.56 \pm 0.17)$. While the latter was reversed by statin treatment, an elevation in blood creatine kinase level indicated the presence of muscle wasting. Fibers from $m$. extensor digitorum longus (EDL) showed significant reduction in cross sectional area in the statin treated groups. Statin treatment also decreased the proliferation and fusion of skeletal myotubes in culture. In line with this, resting intracellular calcium concentration $\left(\left[\mathrm{Ca}^{2+}\right]_{\mathrm{i}}\right)$ was reduced in statin treated satellite cells and myotubes. On the other hand, in adult skeletal muscle fibers statin treatment increased resting $\left[\mathrm{Ca}^{2+}\right]_{\mathrm{i}}(116 \pm 4$ vs. $151 \pm 5 \mathrm{nM} ; \mathrm{n}=33$ and 34$)$ and decreased both twitch and tetanic force both in EDL and $m$. soleus. In addition, in $m$. soleus the duration of twitch and tetanic force was shortened. These results clearly indicate that statin administration in these animals results in a myopathy characterized by decreased muscle force and elevated plasma creatine kinase level.
\end{abstract}

\title{
Keywords
}

Skeletal muscle, cholesterol, statin, force, cell proliferation 


\section{Introduction}

High blood cholesterol level, obesity and atherosclerosis are major health problems. They promote cardiovascular disorders as coronary sclerosis, myocardial ischemia or infarction and stroke. An effective way to decrease the associated morbidity and mortality is to treat the high blood cholesterol level with changes in lifestyle and if that is not sufficient enough, with medications (Merz et al. 2009)

Statins are the most widespread and effective drugs lowering cholesterol levels, claimed to have a number of synergetic effects (Wang et al. 2008). They not only decrease the low density lipoprotein (LDL) and increase the high density lipoprotein (HDL) levels in the blood, but decrease the mortality of cardiovascular diseases (Ward et al. 2007). As of today, about 1.5 million patients' lives were saved by the statin therapy all over the world (Pados and Audikovszky 2007).

The cholesterol lowering effect of statins is exerted by the blockage of the hydroxymethyl-glutaryl-coenzyme-A (HMG-CoA) enzyme thus decreasing the endogenous cholesterol synthesis through the mevalonate pathway (Marcoff and Thompson 2007). This not only decreases the cholesterol production, but the farnesyl pyrophosphate and geranylgeranyl pyrophosphate levels are also lowered, thus the prenylation of proteins is blocked.

Although statins have a lot of beneficial effects, the one year adherence to statin therapy in the USA is less than $50 \%$ (Buettner and Lecker 2008). The main side effect which causes to cease the statin therapy is myopathy: muscle pain, cramps, and weakness, which affects mainly symmetrically the proximal muscles. These complications were observed in $10.5 \%$ of the statin-treated patients, (Bruckert et al. 2005) and claimed to be present for all statins and dose dependent (Thompson et al. 2006). Importantly though blood creatine kinase (CK) level can increase, even independently of muscle complaints. The most feared side effect is, therefore, the fatal rhabdomyolysis characterized by a more than 10 fold increase in 
blood CK level, elevated blood creatinin level and renal failure caused by myoglobinuria (Thompson et al. 2006).

Although the pathomechanism of the statin associated myopathy has been studied in detail and several theories do exist, there is still no real consensus (Buettner and Lecker 2008). The currently prevailing ideas include genetic predisposig factors, mitochondial dysfuction, lack of prenylated proteins and coenzyme Q10, altered cell membrane function and altered calcium homeostasis. One of the major drawbacks for a detailed investigation of the pathomechanism was the lack of an appropriate model system, an animal model in which statin treatment would cause distinct muscle weakness in most of the treated animals not only in one of every several thousand (or million, as in the average human population).

Here we report of an animal model in which increased blood cholesterol levels are present and upon chronic statin treatment muscle degeneration develops in essentially every animal thus enabling the study of statin-induced myopathy. These animals were weaker, showing the pathological effects of statins, including increased blood creatine kinase levels, observed on affected humans. This weakness manifested as a decrease in both twitch and tetanic force of the muscles examined. Together with these alterations the resting intracellular calcium concentration of the fibers was also modified. 


\section{Methods}

\section{Animal models}

Animals were housed in accordance with the guidelines and the approved protocol of the Animal Care Committee of the University of Debrecen. Female Fischer rats were fed with different pre-mixed chows from Charles River Laboratories for at least 21 days, and were allowed to consume the food and tap water ad libitum. The control (C) group was fed with standard rodent chow. For the statin (S) group this standard rodent chow was mixed with $6.4 \mathrm{mg} / \mathrm{kg} / \mathrm{day}$ fluvastatin. The group with high blood cholesterol (hypercholesterolaemic; HC) was fed with high, $5 \mathrm{~g} / \mathrm{kg} /$ day cholesterol containing chow, in addition with $1 \mathrm{~g} / \mathrm{kg} / \mathrm{day}$ cholic acid, which facilitates the absorption of cholesterol in the rat small intestine, and $1 \mathrm{~g} / \mathrm{kg} /$ day thiouracil, which provided us an additional rise in the blood cholesterol level. The hypercholesterolaemic and statin treated $(\mathrm{HC}+\mathrm{S})$ group was kept on high cholesterol and fluvastatin containing diet at the above mentioned concentrations. We set up a second control group, the thiouracil (TU) group, in which the diet contained the same dose of thiouracil as mentioned above, mixed with the standard rodent chow, in order to exclude the effect of hypothyroidism on skeletal muscles. The animals' weights were measured daily together with the daily food intake.

Animals were anaesthetized and sacrificed following a protocol approved by the Animal Care Committee of the University of Debrecen. After pentobarbithal anesthesia (27 mg/kg), the m. extensor digitorum communis (EDC) from the fore limb, and m. extensor digitorum longus (EDL) and $m$. soleus ( $\mathrm{Sol})$ were dissected from the hind limb. Then the abdomen was opened and the abdominal Aorta plunged in order to collect blood samples. Finally the heart was also removed from the animal. 
Some animals were anesthetized with the same dose of pentobarbital, and after collecting the blood from the abdominal Aorta the bodies were used for pathological examination.

\section{Striated muscle cell culture}

Primary striated muscle cell culture was established from 3-7 days old Wistar rats. The animals were sacrificed in accordance with the guidelines and the approved protocol of the Animal Care Committee of the University of Debrecen with a slight modification of a method described in detail elsewhere (Cseri et al. 2002). In brief, the m. quadriceps femoris, $m$. soleus, and $m$. gastrocnaemius were removed, enzymatically digested in a calcium and magnesium free phosphate buffer with $0.75 \mathrm{mg} / \mathrm{ml}$ type 2 collagenase (Sigma, St. Louis, Mo., USA) and trypsin (Difco, Detroit, Mich., USA). Dissociated cells were seeded on glass coverslips in HAM's F12 growth medium (Sigma) containing 15\% fetal bovine serum, and were kept at $37^{\circ} \mathrm{C}$ in a $5 \% \mathrm{CO}_{2}$ containing atmosphere. After 24 hours, two different groups were separated. Control cells were placed in a differentiating medium (DMEM; Sigma) with $5 \%$ Horse serum. The cells in the statin group were placed in the same differentiating medium containing $10 \mathrm{nM}$ fluvastatin.

\section{Blood sample analysis}

The blood cholesterol level of the rats was evaluated to prove the efficacy of the high cholesterol containing diets. We measured the total cholesterol level, the LDL and the HDL cholesterol levels and the triglyceride level. To evaluate the extent of the myopathy the blood CK level was also measured. These tests were performed in the Department of Clinical Biochemistry and Molecular Pathology of the University of Debrecen with their standardized laboratory methods as described earlier (Thomas 1992, Fridewald et al. 1972). 


\section{Pathological tissue examination}

The heart, liver, kidney, EDL and Sol organ weights were measured. In muscles we were looking for signs of myopathy, in the kidneys for signs of renal failure, and in the liver, we examined the fatty degeneration as a result of high blood cholesterol levels.

Immediately after removal, the tissue samples were fixed in $8 \%$ buffered formalin (24 h) and embedded in paraffin wax (Shandon pathcenter, Thermo-Shandon, USA). $4 \mu \mathrm{m}$ sections were cut from paraffin embedded tissue samples and Haematoxillyn-Eosin (H\&E) staining was performed. The samples were investigated with a LEICA DM 2500 microscope equipped with a LEICA DFC 420 camera (Leica Microsystems, Germany). The images were acquired and the diameter of the muscle cross sections were measured using LEICA Application Suite (LAS) V3 software.

\section{Measurement of muscle force}

Muscle contractions were measured as described in our previous reports (Oddoux et al. 2009). In brief, fast and slow twitch muscles, EDL and Sol, were removed and placed horizontally in an experimental chamber continuously superfused $\left(10 \mathrm{ml} \mathrm{min}^{-1}\right)$ with Krebs' solution (containing in $\mathrm{mM}, \mathrm{NaCl} 135, \mathrm{KCl} 5, \mathrm{CaCl}_{2}$ 2.5, $\mathrm{MgSO}_{4} 1$, Hepes 10, glucose 10, $\mathrm{NaHCO}_{3} 10 ; \mathrm{pH} 7.2$; room temperature, $23{ }^{\circ} \mathrm{C}$ ) equilibrated with $95 \% \mathrm{O}_{2}$ plus $5 \% \mathrm{CO}_{2}$. One end of the muscle was attached to a rod while the other to a capacitive mechanoelectric force transducer. Two platinum electrodes placed adjacent to the muscle were used to deliver short, supramaximal pulses of $2 \mathrm{~ms}$ in duration to elicit single twitches. Force responses were digitized at $2 \mathrm{kHz}$ by using Digidata $1200 \mathrm{~A} / \mathrm{D}$ card and stored with Axotape software (Axon Instruments, Foster City, CA, USA). Muscles were then stretched by adjusting the position of 
the transducer to a length that produced the maximal force response and allowed to equilibrate for $60 \mathrm{~min}$.

Single pulses $2 \mathrm{~s}$ apart were used to elicit single twitches. At least 10 twitches were measured under these conditions from every muscle. The individual force transients within such a train varied by less than $3 \%$ in amplitude, thus the mean of the amplitude of all transients was used to characterize the given muscle. To elicit a tetanus, based on the observations of Ranatunga (1982), single pulses were applied with a frequency of $200 \mathrm{~Hz}$ for $200 \mathrm{~ms}$ in case of EDL and with a frequency of $100 \mathrm{~Hz}$ for $300 \mathrm{~ms}$ in case of Sol. Duration of individual twitches and tetani were determined by calculating the time between the onset of the transient and the relaxation to $90 \%$ of maximal force.

To test how muscles fatigue 150 tetani were applied $2 \mathrm{~s}$ apart (Oddoux et al. 2009). The degree of fatigue was expressed by normalizing the amplitude of each tetani to that of the first.

\section{Measurement of resting calcium levels}

Resting intracellular calcium concentration $\left(\left[\mathrm{Ca}^{2+}\right]_{\mathrm{i}}\right)$ was measured both in isolated fibers of the treated adult animals and in primary cultured striated muscle cells.

For the former, EDC was removed and placed into the above described Krebs' solution. The isolation of adult skeletal muscle fibers followed a protocol described previously (Szappanos et al. 2004). In brief, muscle fibers were separated by $90 \mathrm{~min}$ enzymatic digestion at $37^{\circ} \mathrm{C}$ in a shaker water bath with type 2 collagenase (Sigma) containing solution. These fibers were loaded with $5 \mu \mathrm{M}$ Fura-2 AM fluorescent calciumsensitive dye in the presence of $150 \mathrm{nM}$ neostigmin in Krebs' solution. After wash, the dye was excited at two different wavelengths, $340 \mathrm{~nm}$ and $380 \mathrm{~nm}$, using a Deltascan apparatus 
(Photon Technology International, Piscataway, NJ, USA). Emission was detected at $510 \mathrm{~nm}$ and data analysis was performed using a home-made program.

Cells in primary culture were loaded with the same concentration of Fura-2 AM and the loading and measuring process was done exactly the same way as in the adult fibers.

\section{Examination of cell proliferation and differentiation}

Primary cultures of rat striated muscle cells were photographed at 24, 48, and 72 hours after seeding using a Canon EOS-300D (Canon Corp., Japan) digital single lens reflex camera mounted on an inverted microscope. Photos of 5 fields of view per day per culture were taken. Myogenic nuclei were marked manually and morphometric analysis was done on these images. Cell proliferation and differentiation were assessed using the number of nuclei per muscle cell.

\section{Statistical analysis}

Averages - data unless otherwise stated were pooled over the animals or cell cultures within the same group - are presented as mean \pm standard error of the mean. Significance of the differences between control and treated animals was assessed using ZZZ. Cell culture data did not show normal distribution, so Mann-Whitney Rank Sum test was used in that case. Differences were regarded significant at $\mathrm{p}<0.05$. 


\section{Results}

\section{Organ examination}

Although during the autopsy of the rats a slight body weight variability was experienced, the organs were grossly undamaged (Table 1). In microscopic examination of the liver moderate fatty degeneration was found only in rats with high blood cholesterol levels (Fig. 1). We could not detect abnormalities of the cross-striation or any other kind of damage in skeletal muscle samples observed with light microscopy. However, fluctuations in the diameter of EDL muscle fibers were observed on statin treated animals (Fig. 2). We found a significant decrease in the average of cross sectional area of the fibers after statin treatment (from $1835 \pm 79 \mu \mathrm{m}^{2}$ to $1448 \pm 44 \mu \mathrm{m}^{2}$ in the $\mathrm{C}$ and $\mathrm{S}$ groups, $\mathrm{n}=161$ and 288 , respectively; from $2065 \pm 82 \mu \mathrm{m}^{2}$ to $1664 \pm 44 \mu \mathrm{m}^{2}$ in the $\mathrm{HC}$ and $\mathrm{HC}+\mathrm{S}$ groups, $\mathrm{n}=116$ and 226, respectively). Neither the cross sectional area of the fibers from the $\mathrm{HC}$, nor from the TU group $\left(1801 \pm 65 \mu \mathrm{m}^{2}, \mathrm{n}=128\right)$ was significantly different from those of the control.

\section{Blood tests}

Blood cholesterol, triglyceride, and CK levels of the control rats (Table 2) were in the normal range (Suckow et al. 2006, Yerroum et al. 1999). On the other hand, as a result of the diet a marked, slightly more then seven times rise (from 1.45 to 10.7 ) in the total cholesterol level was observed in $\mathrm{HC}$ rats (Table 2). In addition, the ratio of the two main lipoproteins became inverted, while control rats had close to $90 \%$ HDL and only $10 \%$ of the atherogenic LDL, HC rats had only about $40 \%$ of $\mathrm{HDL}$ and the main lipoprotein was the LDL, similar to humans. Although the statin treatment did not change these ratios, it decreased the triglyceride levels in line with earlier reports (Krause and Newton 1995). Most importantly though, while the diet with high cholesterol alone did not elevate the CK level of the rats, 
statin therapy, in the rats with elevated blood cholesterol levels more doubled these values (Table 2), proving the presence of myopathy in the skeletal muscles of the statin treated rats. Note also that while statin treatment in animals with normal blood cholesterol also induced an elevation in blood CK levels, this increase was far less dramatic than that observed in the $\mathrm{HC}+\mathrm{S}$ group.

\section{Effects of statin treatment in cell culture}

After 24 hours of culturing in a medium enhancing proliferation and not containing fluvastatin all cultures reached almost complete confluence (Fig. 3a, d). In the differentiating medium that contained no fluvastatin further 24 hours were enough for muscle cells to fuse and take the spindle shape (Fig. 3b). The apparent decrease in cell count is due to the increase in the number of multinuclear cells. After further 24 hours (72 hours altogether) complete confluence was reached, cells were growing in multiple layers and large myotubes (cells with 10+ nuclei) were present (Fig. 3c). In cultures, where the differentiating medium contained $10 \mathrm{nM}$ fluvastatin the number of spindle-shaped muscle cells clearly dropped by the $48^{\text {th }}$ hour (Fig. 3e). The situation did not visibly improve after further 24 hours of culturing. In contrast to the control group, myotubes were practically absent in the fluvastatin-treated group (Fig. 3f).

Detailed morphometric analysis of the images confirmed the findings based on the visual inspection of the cultures. In the control group the number of myogenic nuclei increased, as compared to the end of the $1^{\text {st }}$ culturing day, by $58 \%$ after 24 hours and by further 54\% after an additional day. In contrast, the number of myogenic nuclei in the fluvastatin-treated group rose by only $3 \%$ after 24 hours and fell by $18 \%$ (decrease not significant) after another 24 hours. The differences in the relative number of nuclei between the control and the statin-treated groups were significant on both days 2 and 3 (Fig. 4a). 
The fusion index showing the percentage of nuclei in multinuclear cells compared to all nuclei showed constant increase in both groups. However, the fusion index was significantly lower on both day 2 (0.68 vs. 0.84$)$ and day 3 (0.81 vs. 0.91$)$ in the fluvastatintreated group than in the control group, respectively (Fig. 4b). The median number of nuclei per cell was also significantly decreased by the statin treatment both after 48 hours (1 vs. 2; statin vs. control, respectively) and 72 hours (1 vs. 3; statin vs. control, respectively) of culturing. These data attest that more than $50 \%$ of muscle cells were mononuclear after 72 hours of culturing in the statin-treated group. The decrease in the median cell size is apparent in the left shift of the histogram depicting data from day 3 (Fig. 4c).

\section{Resting $\mathrm{Ca}^{2+}$ concentration}

In cultured skeletal muscle cells resting $\left[\mathrm{Ca}^{2+}\right]_{\mathrm{i}}$ was significantly decreased after statin treatment both in satellite cells and in myotubes (Fig. 5a). In the latter we found a more than $40 \mathrm{nM}$ decrease in the average value $(119.4 \pm 4.5 \mathrm{nM}$ vs. $72.3 \pm 2.8 \mathrm{nM}$; control vs. statin, respectively). The satellite cells of control cultures had essentially the same resting $\left[\mathrm{Ca}^{2+}\right]_{\mathrm{i}}$ as the myotubes did $(118.9 \pm 3.9 \mathrm{nM})$, while in the statin treated cultures the resting intracellular calcium concentration in the satellite cells was $98.7 \pm 6.2 \mathrm{nM}$.

In contrast, chronic statin treatment in adult animals elevated the resting intracellular calcium level of isolated skeletal muscle fibers (Fig. 5b), independent of the cholesterol levels (85.9 $\pm 1.1 \mathrm{nM}$ vs. $91.3 \pm 1.1 \mathrm{nM}$; in $\mathrm{C}$ vs. $\mathrm{S}$ groups, respectively, and $116.1 \pm 3.8 \mathrm{nM}$ vs. 150.5 $\pm 4.6 \mathrm{nM}$; in $\mathrm{HC}$ vs. $\mathrm{HC}+\mathrm{S}$ groups, respectively). TU treatment alone slightly decreased these values $(75.3 \pm 0.7 \mathrm{nM})$. It is important to stress that although the statin treatment did increase resting $\left[\mathrm{Ca}^{2+}\right]_{\mathrm{i}}$ in animals with normal blood cholesterol, the elevation was much more pronounced in hypercholesterinaemic animals. 


\section{Muscle force}

Figure 6 presents individual traces of twitches (Fig. 6A and B) and tetani (Fig. 6C and D) from both EDL and soleus muscle of TU, $\mathrm{HC}$ and $\mathrm{HC}+\mathrm{S}$ animals. As depicted in Fig. 6 both single twitches and tetani were smaller in amplitude in $\mathrm{HC}+\mathrm{S}$ than in TU and $\mathrm{HC}$ rats. Pooled data for the amplitudes of twitches and tetani are presented in Figure 7A and B, respectively. These reveal significant reduction in twitch force and in maximal force obtained during tetani for both muscle types in $\mathrm{HC}+\mathrm{S}$ muscles compared to $\mathrm{HC}$. This difference was, however, not present between muscles from animals of the TU and $\mathrm{HC}$ groups.

It is important to note here that the cross sectional area of fibers from statin treated animals with high blood cholesterol was smaller than those with similar blood cholesterol but without statin therapy (see above). Force in was thus normalized to cross sectional area and pooled data for $\mathrm{HC}$ and $\mathrm{HC}+\mathrm{S}$ groups were compared. Neither for $\mathrm{EDL}(17.6 \pm 3.1$ vs. $17.2 \pm 3.3 \mathrm{mN} / \mathrm{mm}^{2}$, twitches; $31.5 \pm 5.8$ vs. $34.5 \pm 7.8 \mathrm{mN} / \mathrm{mm}^{2}$, tetani; $\mathrm{HC}$ and $\mathrm{HC}+\mathrm{S}$ animals, respectively) nor for Sol $\left(7.7 \pm 0.7\right.$ vs. $7.0 \pm 0.7 \mathrm{mN} / \mathrm{mm}^{2}$, twitches; $22.5 \pm 2.4$ vs. 20.2 $\pm 4.5 \mathrm{mN} / \mathrm{mm}^{2}$, tetani; $\mathrm{HC}$ and $\mathrm{HC}+\mathrm{S}$ animals, respectively) did normalized values differ significantly between the groups. These results indicate that loss of muscle tissue rather than significant alterations in maximal force of individual fibers was responsible for the decreased force presented in Figs. 6 and 7.

The time course of the transients in Fig. 6 suggests an effect of chronic statin treatment on the kinetics of force production. Fig. 8 thus presents pooled data for the time-to-peak (TTP) and duration of twitches and tetani from both EDL and Sol. Although there were slight variations in TTP values in both EDL and Sol (Fig. 8A and B, respectively) due to the different conditions, these differences did not prove to be significantly significant. On the other hand, the duration of both twitches and tetani in soleus muscles (Fig. 8D) was significantly $(\mathrm{p}<0.05 \mathrm{ZZZ}$, respectively) shorter for the animals which received statin 
treatment $(\mathrm{HC}+\mathrm{S}$ group) than for the animals with high blood cholesterol level (HC group). Similar alterations in the duration of twitches and tetani for EDL - albeit present in tendency (Fig. 8C) - were not statistically significant.

To test how muscles endure fatigue, series of tetani were applied. The ratio of the maximal force at the end of the test and initial tetanus did not show significant reduction in EDL muscles $(0.63 \pm 0.03,0.72 \pm 0.03$, and $0.76 \pm 0.05$ in muscles from $\mathrm{TU}, \mathrm{HC}$, and $\mathrm{HC}+\mathrm{S}$ animals, $n=5,6,8$, respectively). On the other hand, soleus muscles showed significantly greater fatigue in $\mathrm{HC}+\mathrm{S}$ than in either $\mathrm{TU}$ or $\mathrm{HC}$ animals $(0.79 \pm 0.05,0.80 \pm 0.03$, and $0.64 \pm 0.06$ in TU, $\mathrm{HC}$, and $\mathrm{HC}+\mathrm{S}$ muscles, $\mathrm{n}=7,6,9$, respectively). 


\section{Discussion}

Here we report of an animal model in which the administration of cholesterol together with cholic acid and thiouracyl in the diet resulted in a significant elevation of the blood cholesterol levels. In these animals the subsequent chronic administration of fluvastatin resulted in a clear myopathy described by a reduction in fiber cross sectional area and in muscle force. Not only was peak twitch and tetanic force reduced in both fast $-m$. EDL - and in slow $-m$. soleus - muscles, their duration in $m$. soleus was also decreased. In addition, resting $\left[\mathrm{Ca}^{2+}\right]_{i}$ was elevated in muscle fibers isolated from animals with high blood cholesterol and treated with statins. It should be noted that chronic statin treatment alone, that is in animals kept on normal rodent chow and having normal blood cholesterol, did not produce these effects. On the other hand, the presence of fluvastatin in the culture medium reduced the proliferation and prolonged the differentiation process of cultured myocytes.

It is important to stress that we used artificially hypothyroid animals to further elevate blood cholesterol levels. This hypothyroid status of the animals with high blood cholesterol already resulted in an altered muscle function, and - combined with statin therapy - it manifested in a marked myopathy. To exclude the possibility that the reduction in muscle force was due to the hypothyroidism - caused by the thiouracyl in the diet - alone, animals kept on a thiouracyl diet were used as reference. Our results clearly demonstrated that the severe muscle problems were associated with the administration of fluvastatin and not with the hypothyroid state of the animals.

Alterations in the calcium homeostasis were also suggested to play an important role in the pathophysiology of statin-induced myopathy (e.g. Sacher et al. 2005). A structural weakness in the T-tubular system, as T-tubules are more vulnerable to cholesterol depletion than other parts of the sarcolemma or the sarcoplasmic reticulum, of statin-treated patients has been demonstrated (Draeger et al. 2006). Furthermore, altered $\mathrm{Ca}^{2+}$ spark activity has been 
reported following the acute treatment of muscle fibers by simvastatin (Sirvent et al. 2005). In addition, ryanodine receptor type 3 (RyR3) has been shown to be up-regulated in patients with statin-associated myopathy (Draeger et al. 2010). Since RyR3 is more sensitive to activating $\mathrm{Ca}^{2+}$, its overexpression in striated muscle is accompanied an increase in resting $\left[\mathrm{Ca}^{2+}\right]_{\mathrm{i}}$ (Legrand et al. 2008). In line with the above, we observed a distinct elevation in the resting cytosolic levels of $\mathrm{Ca}^{2+}$ in muscle fibers following chronic statin treatment in this study.

The cause of statin-associated myopathy is not yet clear, although there are some pharmacological properties, which have been characterized. Statin-induced muscle symptoms are dose-dependent and seem to be present for every molecule in the drug family. The risk factors are reported to be the old age, renal or hepatic impairment, concomitant use of fibrates, and co-administered drugs that either metabolize in the liver on the same cytochrome P450 (CYP) enzyme, or inhibit the CYP enzyme (CYP2C9 for fluvastatin, CYP3A4 for other statins; e.g. macrolide antibiotics, antifungal medications, gemfibrozil, cyclosporine, and grapefruit juice). These latter drugs can increase the serum concentration of statins, leading to higher risk of myopathy. These explain why several studies (e.g. Obata et al. 2009) as well as ours used higher statin doses than that present in therapy.

The statin-associated myopathy is reported to be more common (9-20\% of statin users) than indicated in the clinical studies (1-5\%), and often leads to stop the drug taking (50\% after 1 year of treatment), thus these patients loose the potential beneficial effects of statin therapy on the blood cholesterol, cardiovascular morbidity and mortality (Buettner and Lecker 2008). Even in patients without myopathic complaints, statin therapy induced ultrastructural skeletal muscle damage (Draeger et al. 2006).

Many potential mechanisms underlying the statin induced myopathy that have been reported in the literature (see e.g. Sirvent et al. 2008) could explain the alterations - modified 
resting $\left[\mathrm{Ca}^{2+}\right]_{\mathrm{i}}$, reduced muscle force, hindered differentiation of myotubes - seen in this study. These include decreased cholesterol production leading to cholesterol depletion in the myocyte membranes (Harper and Jacobson 2007), and blocked HMG-CoA reductase activity resulting in decreased prenylation and thus altered function of small GTP-ases (Wang et al. 2007). Normal cholesterol levels in phospholipids bilayers seem important in reducing $\mathrm{Na}^{+}$ leakage through these membranes and, therefore, lowering the energy expenditure of the $\mathrm{Na}^{+}-$ $\mathrm{K}^{+}$-pump and thus preventing ATP depletion of the cell (Haines 2001). Reduced amounts of cholesterol in the surface membrane would then lead to ATP depletion and, therefore, altered calcium homeostasis. Statins have also been reported to induce mitochondrial dysfunction (Sirvent et al. 2005), and/or Coenzyme Q10 deficiency, which can also lead to myopathy (Harper and Jacobson 2007, Ghirlanda et al. 1993) since these mechanisms tend to activate the apoptotic cascade (Dirks and Jones 2006, Kaufmann et al. 2006, Kohro and Yamazaki 2009). Furthermore, changes in the ubiquitin-proteasome pathway have been referred to be associated with statin-induced myopathy as well (Buettner and Lecker 2008).

Hypothyroidism or inherited mitochondrial dysfunction alone may lead to myopathy, thus worsen the side effect of statins. It should also be noted that statin treatment in control animals (group C) resulted in changes in the same direction albeit the changes were smaller (Buettner and Lecker 2008). Nevertheless, hypothyroidism does seem to play an important part in the statin-associated myopathy. In a case report, an asymptomatic hypothyroid patient received statin therapy and observed lower extremity myalgia within 4 weeks of treatment, which resolved ceasing the statin therapy (Bar et al. 2007). Another, more serious case was reported revealing a chronic, high dose grapefruit juice consumption triggered statinassociated rhabdomyolysis with a CK elevated to 12640 U/l. The patient observed bilateral proximal leg weakness (Dreier and Endres 2004). 
In line with the above mentioned observations we found a clear reduction in muscle strength and signs of muscle wasting in our animals with high blood cholesterol, thus treated with thiouracyl, following chronic statin administration. These animals not only had increased levels of plasma CK but displayed reduced twitch and tetanic force. In addition, we found a reduced ability of cultured myoblasts to differentiate, fuse and form multinucleated myotubes suggesting that the damaged muscle would also have an impaired repair mechanism. Our results thus raise the possibility that a hypothyroid status in hypercholesterolaemia increases the incidence of severe rhabdomyolysis if chronic statin treatment is applied. Furthermore, the animal model presented here should serve as a useful tool in studies on statin-induced myopathies. 


\section{Acknowledgements}

The authors wish to thank R. Öri for the excellent technical assistance. This work was supported by grants from the Hungarian Scientific Research Found (OTKA K-75604, NK78398), from the Hungarian Ministry of Health (ETT 186/2009) and from the Hungarian Ministry of Education (K-2009-TÁMOP-4.2.2-08/1/2008-0019). 


\section{References}

Bar SL, Holmes DT, Frohlich J (2007) Asymptomatic hypothroidism and statin-induced myopathy. Can Fam Physician 53(3):428-431.

Bruckert E, Hayem G, Dejager S, Yau C, Bégaud B (2005) Mild to Moderate Muscular Symptoms with High-Dosage Statin Therapy in Hpyerlipidemic Patients - The PRIMO Study. Cardiovasc Drugs Ther 19(6):403-414.

Buettner C, Lecker SH (2008) Molecular basis for statin-induced muscle toxicity: implications and possibilities. Pharmacogenomics 9(8):1133-1142.

Cseri J, Szappanos H, Szigeti GP, Csernátony Z, Kovács L, Csernoch L (2002) A purinergic signal transduction pathway in mammalian skeletal muscle cells in culture. Pflügers Archiv 443:731-738.

Dirks AJ, Jones KM (2006) Statin-induced apoptosis and skeletal myopathy. Am J Physiol Cell Physiol 291(6):C1208-C1212.

Draeger A, Monastyrskaya K, Mohaupt M, Hoppeler H, Savolainen H, Allemann C, Babiychuk EB (2006) Statin therapy induces ultrastructural damage in skeletal muscle in patients without myalgia. J Pathol 210(1):94-102.

Draeger A, Sanchez-Freire V, Monastyrskaya K, Hoppeler H, Mueller M, Breil F, Mohaupt MG, Babiychuk EB (2010) Statin therapy and the expression of genes that regulate calcium homeostasis and membrane repair in skeletal muscle. Am J Pathol 177(1):291-299.

Dreier JP, Endres M (2004) Statin-associated rhabdomyolysis triggered by grapefruit consumption. Neurology 62(4):670. 
Fridewald WF, Levy RI, Frederickson DS (1972) Estimation of LDL-Cholesterol concentration without use of the preparative ultra-centrifuge. Clin Chem 18:499-502.

Ghirlanda G, Oradei A, Manto A, Lippa S, Uccioli L, Caputo S, Greco AV, Littarru GP (1993) Evidence of plasma CoQ10-lowering effect by HMG-CoA reductase inhibitors: a double-blind, placebo-controlled study. J Clin Pharmacol 33:226-229.

Haines TH (2001) Do sterols reduce proton and sodium leaks through lipid bilayers? Prog Lipid Res 40:299-324.

Harper CR, Jacobson TA (2007) The broad spectrum of statin myopathy: from myalgia to rhabdomyolysis. Curr Opin Lipidol 18(4):401-408.

Kaufmann P, Török M, Zahno A, Waldhauser KM, Brecht K, Krähenbühl S (2006) Toxicity of statins on rat skeletal muscle mitochondria. Cell Mol Life Sci 63(19-20):2415-2425.

Kohro T, Yamazaki T (2009) Mechanism of statin-induced myopathy investigated using microarray technology. J Atheroscler Thromb 16(1):30-32.

Krause BR, Newton RS (1995) Lipid-lowering activity of atorvastatin and lovastatin in rodent species: triglyceride-lowering in rats correlates with efficacy in LDL animal models. Atherosclerosis 117(2):237-244.

Legrand C, Giacomello E, Berthier C, Allard B, Sorrentino V, Jacquemond V (2008) Spontaneous and voltage-activated $\mathrm{Ca}^{2+}$ release in adult mouse skeletal muscle fibres expressing the type 3 ryanodine receptor. J Physiol 586(2):441-457.

Marcoff L, Thompson PD (2007) The role of coenzyme Q10 in statin-associated myopathy: a systematic review. J Am Coll Cardiol 49:2231-2237. doi:10.1016/j.jacc.2007.02.049 
Merz CNB, Alberts MJ, Balady GJ, Ballantyne CM et al (2009) ACCF/AHA/ACP 2009

Competence and Training Statement: A Curriculum on Prevention of Cardiovascular Disease

A Report of the American College of Cardiology Foundation/American Heart Association/American College of Physicians Task Force on Competence and Training (Writing Committee to Develop a Competence and Training Statement on Prevention of Cardiovascular Disease). Circulation 120:e100-e126.

Oddoux S, Brocard J, Schweitzer A, Szentesi P, Giannesini B, Brocard J, Fauré J, PernetGallay K, Bendahan D, Lunardi J, Csernoch L, Marty I. (2009) Triadin deletion induces impaired skeletal muscle function. J Biol Chem 284(50):34918-34929.

Pados Gy, Audikovszky M (2007) A statinkezelés biztonságossága. Hyppocrates ISSN 14193337. 9(1):20-25.

Sacher J, Weigl L, Werner M, Szegedi C, Hohenegger M (2005) Delineation of myotoxicity induced by 3-hydroxy-3-methylglutaryl CoA reductase inhibitors in human skeletal muscle cells. J Pharmacol Exp Ther 314(3):1032-1041.

Sirvent P, Mercier J, Vassort G, Lacampagne A (2005) Simvastatin triggers mitochondriainduced $\mathrm{Ca}^{2+}$ signaling alteration in skeletal muscle. Biochem Biophys Res Commun 329(3):1067-1075.

Sirvent P, Mercier J, Lacampagne A (2008) New insights into mechanisms of statinassociated myotoxicity. Curr Opin Pharmacol 8(3):333-338.

Suckow MA, Weisbroth SH, Franklin CL (2006) The Laboratory Rat. Elsevier Inc. ISBN: 978-0-12-074903-4. 
Szappanos H, Cseri J, Deli T, Kovács L, Csernoch L. (2004) Determination of depolarisationand agonist-evoked calcium fluxes on skeletal muscle cells in primary culture. $\mathrm{J}$ Biochem Biophys Methods 59(1):89-101.

Thomas L Labor und diagnose (1992) Labor und Diagnose: Die Medizinische Verlagsgesellschaft. 4th ed. Marburg:

Thompson PD, Clarkson PM, Rosenson RS; National Lipid Association Statin Safety Task Force Muscle Safety Expert Panel (2006) An assessment of statin safety by muscle experts. Am J Cardiol 97(8A):69C-76C.

Yerroum M, Braconnier F, Chariot P (1999) Influence of handling procedures on rat plasma creatine kinase activity. Muscle Nerve 22(8):1119-1121.

Wang CY, Liu PY, Liao JK (2008) Pleiotropic effects of statin therapy: molecular mechanisms and clinical results. Trends Mol Med 14(1):37-44.

Ward S, Lloyd Jones M, Pandor A, Holmes M, Ara R, Ryan A, Yeo W, Payne M (2007) A systematic review and economic evaluation of statins for the prevention of coronary events. Health Technol Assess. 11(14):1-160. iii-iv. 


\section{Figure legends}

Figure 1. Intact liver tissue structure observed with light microscope $(C, S, H C+S, T U)$. Moderate (small \& large droplets, marked with arrows in the inset) fatty deposition seen in hypercholesterolaemic (HC) rats. H\&E staining at 20x magnification.

Figure 2. Pathologic structural changes cannot be seen on the cross-section of EDL muscle fibers under light microscope at any sample. However, fluctuation of the diameter of EDL muscle fibers can be observed on the statin treated samples. H\&E staining at 40x magnification.

Figure 3. Representative images of control (A-C) and statin-treated (D-F) rat primary skeletal muscle cell cultures. Fluvastatin $(10 \mathrm{nM})$ was applied after the $24^{\text {th }}$ hour of culturing. Arrows on panel $\mathrm{C}$ denote large myotubules with more than 10 nuclei.

Figure 4. Detailed morphometric data of the effect of fluvastatin treatment on the proliferation and differentiation of cultured skeletal muscle cells. (A) Relative number of myogenic nuclei compared to the data at the $24^{\text {th }}$ hour (the beginning of drug treatment in group S). (B) Fusion index showing the ratio of myogenic nuclei in multinuclear cells compared to all myogenic nuclei. (C) Histogram showing the distribution of cell size expressed as the number of nuclei in the cell. Error bars represent SEM. Asterisks denote significant difference between $\mathrm{C}$ and $\mathrm{S}$ treated cell cultures $(\mathrm{p}<0.05)$.

Figure 5. (A) Resting $\mathrm{Ca}^{2+}$ concentration of satellite cells and myotubes in primary rat skeletal muscle culture. In control cultures satellite cells $\mathrm{N}=37$, myotubes $\mathrm{N}=43$. In statin treated cultures satellite cells $\mathrm{N}=13$, myotubes $\mathrm{N}=12$. (B) Resting $\mathrm{Ca}^{2+}$ concentration of the isolated myofibers of rats kept on different diets. Numbers in each column represent number of myofibers. Error bars represent SEM. Asterisks denote significant difference from C, \# from TU and \& from $\mathrm{HC}$ rats, $(\mathrm{p}<0.05)$. 
Figure 6. Force transients in isolated muscles of $\mathrm{TU}, \mathrm{HC}$ and $\mathrm{HC}+\mathrm{S}$ rats. $(\mathrm{A}-\mathrm{B})$ Single twitches elicited by short (2 ms), supramaximal (5 V) stimulation on EDL (A) and Soleus (B) from $\mathrm{TU}$ (dotted), $\mathrm{HC}$ (dashed line) and $\mathrm{HC}+\mathrm{S}$ (continuous line) rats. $(\mathrm{C}-\mathrm{D})$ Tetani elicited by a train of supramaximal stimulation at $200 \mathrm{~Hz}$ for $200 \mathrm{~ms}$ on EDL (C) and at $100 \mathrm{~Hz}$ for $300 \mathrm{~ms}$ on soleus muscle (D) from TU (dotted), $\mathrm{HC}$ (dashed line), and $\mathrm{HC}+\mathrm{S}$ (continuous line) rats. Measurements were made at $23{ }^{\circ} \mathrm{C}$.

Figure 7. Average twitch and maximal force. Data were pooled from 5 TU, $6 \mathrm{HC}$, and 10 $\mathrm{HC}+\mathrm{S}$ rats, after averaging the maximum of the force transients over each individual record (usually 10) measured on an individual muscle. Average amplitude of the twitches (A) and of the tetani (B). Asterisks denote significant difference between $\mathrm{HC}$ and $\mathrm{HC}+\mathrm{S}$ rats $(\mathrm{p}<0.05)$. 
Table 1. Average of the body weight and the weights of different organs in control and statin treated rats.

\begin{tabular}{|l|c|c|c|c|}
\hline \multicolumn{1}{|c|}{ Animal } & Body weight & Heart & m. EDL & m. soleus \\
$(\mathrm{g})$ & $(\mathrm{mg})$ & $(\mathrm{mg})$ & $(\mathrm{mg})$ \\
\hline $\mathrm{C}(22)$ & $127.3 \pm 20.6$ & $527 \pm 34$ & $76 \pm 3$ & $91 \pm 7$ \\
\hline TU (10) & $134.1 \pm 8.6$ & $354 \pm 21$ & $57 \pm 3^{*}$ & $72 \pm 3^{*}$ \\
\hline HC (14) & $119.3 \pm 2.1^{\#}$ & $356 \pm 27$ & $51 \pm 3$ & $68 \pm 3$ \\
\hline HC+S (20) & $132.3 \pm 3.6^{\$}$ & $358 \pm 36$ & $55 \pm 4$ & $74 \pm 7$ \\
\hline
\end{tabular}

Number in brackets represents the number of animals. ${ }^{*}$ denotes significant difference from C, ${ }^{\#}$ denotes significant difference from TU and ${ }^{\$}$ denotes significant difference from HC. Note that since $\mathrm{HC}$ and $\mathrm{HC}+\mathrm{S}$ animals have thiouracyl in their diet comparison in these cases were only made to the TU group. 
Table 2. The average level of total cholesterol, triglyceride, the creatine kinase and the ratio of HDL and LDL cholesterol in the blood of the rats.

\begin{tabular}{|l|c|c|c|c|}
\hline Animal group & $\begin{array}{c}\text { Cholesterol } \\
(\mathrm{mmol} / \mathrm{L})\end{array}$ & $\begin{array}{c}\text { Triglyceride } \\
(\mathrm{mmol} / \mathrm{L})\end{array}$ & $\begin{array}{c}\text { Creatine Kinase } \\
(\mathrm{U} / \mathrm{L})\end{array}$ & HDL/LDL \\
\hline $\mathrm{C}(15)$ & $1.52 \pm 0.05$ & $0.74 \pm 0.08$ & $636.3 \pm 139.2$ & $9.95 \pm 1.79$ \\
\hline $\mathrm{S}(10)$ & $1.58 \pm 0.10$ & $0.56 \pm 0.06$ & $959.6 \pm 361.0$ & $6.57 \pm 0.94$ \\
\hline TU (9) & $1.45 \pm 0.08$ & $0.35 \pm 0.04^{*}$ & $563.9 \pm 136.4$ & $3.44 \pm 0.59^{*}$ \\
\hline HC (16) & $10.74 \pm 20^{\#}$ & $0.53 \pm 0.06^{\#}$ & $576.3 \pm 149.2$ & $0.64 \pm 0.07^{\#}$ \\
\hline HC+S (12) & $6.81 \pm 1.69^{\#}$ & $0.46 \pm 0.06$ & $1513.4 \pm 410.3^{\# \$}$ & $0.94 \pm 0.22^{\#}$ \\
\hline
\end{tabular}

Numbers in brackets represent the number of animals. ${ }^{*}$ denotes significant difference from C, ${ }^{\#}$ denotes significant difference from TU and ${ }^{\$}$ denotes significant difference from HC. Note that since $\mathrm{HC}$ and $\mathrm{HC}+\mathrm{S}$ animals have thiouracyl in their diet comparison in these cases were only made to the TU group. 